Doi: HTTPS://DOI.ORG/10.23910/IJEP/2019.6.3.0316

\title{
Variability in Morphological and Phenological Characters of Apricot (Prunus armeniaca L.) Genotypes from Wild Population in Shimla District of Himachal Pradesh
}

\author{
Nirmla Chauhan*, Dinesh Singh, K. Kumar, R. K. Dogra and Suman Bodh
}

Dept. of Fruit Science, Dr Y. S. Parmar University of Horticulture and Forestry, Nauni, Solan, H.P. (173 230), India

\author{
Corresponding Author \\ Nirmla Chauhan \\ e-mail: nirmlachauhan90@gmail.com
}

\author{
Article History \\ Article ID: IJEP0316 \\ Received in $23^{\text {rd }}$ July, 2019 \\ Received in revised form 06 ${ }^{\text {th }}$ August, 2019 \\ Accepted in final form $14^{\text {th }}$ August, 2019
}

\begin{abstract}
Apricot (Prunus armeniaca L.) is one of the most important stone fruits, basically due to its good taste and multiple uses. Commercial growing of apricots is marred by monoculture owing to predominance of very few cultivars like New Castle, Royal etc. However, there is a wide array of germplasm of apricot growing wild in north-western Himalayas. The ever-changing environment and consumer preferences demand apricots with wider adaptation and better yield and fruit quality. This is necessitated more from crop diversification point of view, and also in view of the widespread use of apricot in processing, thus enhancing farmer's income. So, efforts were made to select superior genotypes from wild apricots in Shimla district of Himachal Pradesh during 2015-16. Observations on flowering, tree and leaf characters were recorded in 42 genotypes. Late and extended flowering was observed among the selected genotypes. Maximum trunk girth (78.84 $\mathrm{cm}$ ) was recorded in SCK5 under Chirgaon location. Opening of flower stated from $4^{\text {th }}$ week of February (SRB1, SRB3, SRB4, SR2, STB1, STB2 and STB3) and extended upto $1^{\text {st }}$ week of April (SCK1, SCK3, SCK4, SCK5 and SCK6). These characters can be used for further breeding improvement of apricots like late flowering trait to protect it from spring frost.
\end{abstract}

Keywords: Genotypes, flowering, selection, Shimla, wild apricot

\section{Introduction}

Apricot (Prunus armeniaca L.) is a deciduous tree species belonging to family Rosaceae and grown worldwide in mild temperate to extreme cold regions. In India, it is cultivated in hilly regions of Himachal Pradesh, Jammu and Kashmir, Uttarakhand (Parmar and Kaushal, 1982). Some varieties of apricot can be grown in slightly warmer regions of Punjab, Haryana and plains of Himachal Pradesh. Total area under apricot cultivation in Himachal Pradesh is 3680 hectares with production 4635 MT during 2017-2018 (Department of Horticulture, 2019). This delicious stone fruit is commercially used both for dessert and processing purpose and it is rich in vitamin A and contains more carbohydrates, protein, phosphorous and niacin than majority of other common fruits (Teskey and Shoemaker, 1972).

Besides the existing commercial plantations of cultivated forms, there is prevalence of large populations of seedling apricot trees growing wild commonly known as 'Zardalu', 'Chulli', 'Chir' or 'Sara' found naturalized in the north-western Himalayas particularly in Kinnaur, Lahaul and Spiti, Chamba, Kullu, Shimla, Solan and Mandi districts of Himachal Pradesh. The natural population of wild seedling apricot found growing as scattered trees in the interiors of dry temperate region of Himachal Pradesh are particularly important for high sugar content (drying type). The kernels of wild apricots are both sweet and bitter and the bitter kernels are used for oil extraction. In the temperate regions of Himachal Pradesh, large quantities of these wild apricots are collected for oil extraction.

It is well known that there is a wide array of germplasm of wild apricot growing in north-western Himalayas. The wild apricot has also been collected in past but that has been used mainly as rootstock for cultivated types. Hitherto, a very meager effort has been made by the horticulturists for the selection of superior apricot genotypes with desirable traits from these natural populations present abundantly in Himachal Pradesh. There may be some seedling trees growing wild which may have very good dessert quality, high sugar content and sweet kernels and suitable for drying. Dry apricot has tremendous export potential provided international standards are maintained in production and marketing technology and this only can be achieved through the planting of quality material commercially having desirable traits. So keeping in view the present investigations were carried out in Shimla district of Himachal Pradesh to select superior genotypes with late flowering characters from wild population. 


\section{Materials and Methods}

The study was conducted covering existing seedling apricot tree population in Shimla district of Himachal Pradesh. The region opted for research particularly falls under high hills wet temperate zone which stretched from N $31^{\circ} 07.779^{\prime}$ to $\mathrm{N}$ $31^{\circ} 44.338^{\prime}$ and $\mathrm{E} 077^{\circ} 15.810^{\prime}$ to $\mathrm{E} 077^{\circ} 61.363^{\prime}$ with elevation ranging from 1411-2535 meters above mean sea level. The selection was based on fruit size and TSS as well as feedback collected (estimated age of the tree, sweet kernel, high oil content and attractive fruit colour with red blush) from local people and farmers in the form of questionnaire. On the basis of pre-selection survey about 250 genotypes were selected, out of which 42 genotypes were taken for further analysis and their tree, flowering, and foliage characters were observed (Table 1).

The experimental data of all the metric characters studied were subjected to the statistical analysis. Test of significance suggested by Panse and Sukhatme (1985) was used. The statistical analysis was carried out for each observed character by using MS-Excel.

\begin{tabular}{llc}
\hline \multicolumn{3}{l}{ Table 1: Location details of apricot accessions selected from } \\
Shimla district & \multicolumn{2}{l}{ Code } \\
\hline Location & Village & SRB (1-6) \\
\hline Rampur -R & Bajetli -B & SR (1-4) \\
Rampur -R & Rampur & SJM (1-4) \\
Jubbal -J & Mandhol-M & SROB (1) \\
Rohru -RO & Baggi -B & SCJ (1-3) \\
Chirgaon-C & Jhainari-J & SCD (1) \\
& Denwari -D & SCDH (1-2) \\
& Dhamwari- DH & SCK (1-7) \\
& Khaniyara -K & SM (1-3) \\
Mashobra -M & Mashobra & SKK (1-4) \\
Kotkhai -K & Kyari-K & SKUM (1-4) \\
Kumarsain -KU & Madhwani -M & STB (1-3) \\
Theog-T & Balag -B &
\end{tabular}

\section{Results and Discussion}

Tree vigour and growth habit are important horticultural trait which determines the bearing age and potential in fruit crops. The apricot trees under study varied in age ranging from 15 years to more than 45 years.

Among the genotypes studied from Shimla district 28 genotypes had shown upright growth habit, while 14 genotypes were found to be spreading type. Trunk girth ranged from 18.72 to $78.84 \mathrm{~cm}$ in the genotypes of Shimla district. Maximum trunk girth $(78.84 \mathrm{~cm})$ was observed in SCK5 and minimum $(18.72 \mathrm{~cm})$ in SKK3. Average trunk girth was reported to be $47.53 \mathrm{~cm}$ and their coefficient of variation was $33.77 \%$. The shoot colour of current growth was observed to be Green-Group (143 A) in 8 genotypes; (143 B) in 4 genotypes; (143 C) in 6 genotypes; (142 A) in 11 genotypes, (142 B) in 5 genotypes and (141 B) in 8 genotypes. In case of one year old shoot, genotypes were found to be Grey Orange Group (166 A) in 8 genotypes; (166 B) in 4 genotypes; (165 A) in 19 genotypes and (165 B) in 11 genotypes among the genotypes studied from Shimla district (Table 2).

Table 2: Tree characters of seedling apricot genotypes selected from Shimla district

\begin{tabular}{|c|c|c|c|c|}
\hline \multirow{2}{*}{$\begin{array}{l}\text { Geno- } \\
\text { type }\end{array}$} & \multirow{2}{*}{$\begin{array}{l}\text { Growth } \\
\text { habit }\end{array}$} & \multirow{2}{*}{$\begin{array}{l}\text { Trunk } \\
\text { girth } \\
(\mathrm{cm})\end{array}$} & \multicolumn{2}{|c|}{ Shoot colour } \\
\hline & & & $\begin{array}{l}\text { Current } \\
\text { growth }\end{array}$ & $\begin{array}{l}\text { One year } \\
\text { old shoot }\end{array}$ \\
\hline SRB1 & Upright & 64.85 & ${ }^{*}$ GG-143 C & ${ }^{* *} \mathrm{GO}-165 \mathrm{~A}$ \\
\hline SRB2 & Upright & 51.96 & GG-143 C & GO-166 B \\
\hline SRB3 & Upright & 45.45 & GG-143 B & GO-165 A \\
\hline SRB4 & Spreading & 53.86 & GG-142 A & GO-165 A \\
\hline SRB5 & Spreading & 56.09 & GG-141 B & GO-165 A \\
\hline SRB6 & Upright & 60.47 & GG-141 B & GO-165 B \\
\hline SR1 & Upright & 64.97 & GG-143 C & GO-165 B \\
\hline SR2 & Upright & 32.14 & GG-142 B & GO-165 A \\
\hline SR3 & Upright & 48.66 & GG-143 C & GO-165 A \\
\hline SR4 & Upright & 34.55 & GG-143 A & GO-165 A \\
\hline SJM1 & Spreading & 26.58 & GG-142 B & GO-165 A \\
\hline SJM2 & Spreading & 30.73 & GG-143 C & GO-166 B \\
\hline SJM3 & Upright & 47.29 & GG-142 B & GO-165 A \\
\hline SJM4 & Spreading & 31.54 & GG-142 A & GO-165 A \\
\hline SROB1 & Upright & 18.86 & GG-141 B & GO-165 A \\
\hline SCJ1 & Upright & 31.46 & GG-143 A & GO-165 A \\
\hline $\mathrm{SCJ} 2$ & Upright & 54.50 & GG-143 A & GO-165 A \\
\hline $\mathrm{SCJ} 3$ & Upright & 64.60 & GG-143 A & GO-165 B \\
\hline SCD1 & Spreading & 70.68 & GG-142 A & GO-165 B \\
\hline $\mathrm{SCDH} 1$ & Upright & 33.75 & GG-141 B & GO-165 A \\
\hline $\mathrm{SCDH} 2$ & Upright & 43.86 & GG-142 A & GO-166 A \\
\hline SCK1 & Upright & 32.30 & GG-142 A & GO-165 B \\
\hline SCK2 & Spreading & 27.74 & GG-143 B & GO-165 B \\
\hline SCK3 & Spreading & 30.90 & GG-143 A & GO-165 B \\
\hline SCK4 & Upright & 78.00 & GG-142 A & GO-165 B \\
\hline SCK5 & Upright & 78.84 & GG- $141 \mathrm{~B}$ & GO-166 B \\
\hline SCK6 & Upright & 64.36 & GG-142 A & GO-166 A \\
\hline SCK7 & Upright & 61.47 & GG-142 A & GO-165 A \\
\hline SM1 & Upright & 43.54 & GG-143 A & GO-165 B \\
\hline SM2 & Spreading & 50.36 & GG-141 B & GO-166 A \\
\hline SM3 & Upright & 36.34 & GG-141 B & GO-166 A \\
\hline SKK1 & Upright & 77.46 & GG-143 B & GO-165 B \\
\hline
\end{tabular}

Table 2: Continue... 


\begin{tabular}{|c|c|c|c|c|c|c|c|c|c|c|}
\hline \multirow{3}{*}{$\begin{array}{l}\text { Geno- } \\
\text { type }\end{array}$} & \multirow{3}{*}{$\begin{array}{l}\text { Growth } \\
\text { habit }\end{array}$} & \multirow{3}{*}{$\begin{array}{l}\text { Trunk } \\
\text { girth }(\mathrm{cm})\end{array}$} & \multicolumn{2}{|c|}{ Shoot colour } & \multirow{2}{*}{\multicolumn{6}{|c|}{$\begin{array}{l}\text { Table 3: Foliage characters of apricot accessions selected } \\
\text { from Shimla district }\end{array}$}} \\
\hline & & & \multirow{2}{*}{$\begin{array}{l}\text { Current } \\
\text { growth }\end{array}$} & \multirow{2}{*}{$\begin{array}{l}\text { One year } \\
\text { old shoot }\end{array}$} & & & & & & \\
\hline & & & & & \multirow[t]{3}{*}{ Genotype } & \multirow{3}{*}{$\begin{array}{l}\text { Leaf } \\
\text { length } \\
(\mathrm{cm})\end{array}$} & \multirow{2}{*}{$\begin{array}{l}\text { Leaf } \\
\text { width } \\
(\mathrm{cm})\end{array}$} & \multirow{2}{*}{$\begin{array}{l}\text { Leaf } \\
\text { area } \\
\left(\mathrm{cm}^{2}\right)\end{array}$} & \multirow{3}{*}{$\begin{array}{l}\text { Petiole } \\
\text { length } \\
(\mathrm{mm})\end{array}$} & \multirow{2}{*}{$\begin{array}{l}\text { No. of } \\
\text { glands }\end{array}$} \\
\hline SKK2 & Upright & 26.02 & GG-143 A & GO-166 A & & & & & & \\
\hline SKK3 & Upright & 18.72 & GG-143 A & GO-165 A & & & \multirow{2}{*}{2.61} & \multirow{2}{*}{36.94} & & \multirow{2}{*}{2.33} \\
\hline SKK4 & Upright & 50.13 & GG-142 B & GO-165 A & SRB1 & 4.14 & & & 38.13 & \\
\hline SKUM1 & Spreading & 35.81 & GG-142 B & GO-166 A & \multirow{2}{*}{ SRB2 } & \multirow{2}{*}{4.06} & 2.52 & 33.55 & 40.11 & 3.33 \\
\hline SKUM2 & Upright & 61.77 & GG-141 B & GO-166 B & & & 2.63 & 32.26 & 37.40 & 1.67 \\
\hline SKUM3 & Spreading & 55.24 & GG-142 A & GO-165 B & SRB4 & 4.74 & 2.61 & 33.13 & 38.11 & 2.00 \\
\hline SKUM4 & Spreading & 44.95 & GG-143 B & GO-166 A & SRB5 & 4.43 & 1.88 & 33.88 & 45.70 & 3.00 \\
\hline STB1 & Upright & 52.55 & GG-142 A & GO-166 A & SRB6 & 5.56 & 3.95 & 39.09 & 36.85 & 1.00 \\
\hline STB2 & Spreading & 48.57 & GG-143 C & Go-165 A & SR1 & 2.98 & 1.96 & 29.17 & 39.05 & 0.00 \\
\hline STB3 & Spreading & 54.26 & GG-142 A & GO-165 A & SR2 & 4.86 & 3.51 & 31.71 & 30.13 & 4.00 \\
\hline Range & & 18.72- & & & SR3 & 2.54 & 3.72 & 26.52 & 36.65 & 2.67 \\
\hline & & 78.84 & & & SR4 & 4.02 & 2.16 & 32.55 & 29.05 & 1.67 \\
\hline Mean & & 47.53 & & & SJM1 & 3.86 & 2.68 & 33.51 & 22.56 & 0.00 \\
\hline SE \pm & & 2.48 & & & SJM2 & 3.57 & 2.11 & 33.96 & 26.82 & 4.00 \\
\hline SD & & 16.05 & & & SJM3 & 3.32 & 1.74 & 32.31 & 30.33 & 3.33 \\
\hline CV (\%) & & 33.77 & & & SJM4 & 3.65 & 2.86 & 29.01 & 27.75 & 2.33 \\
\hline *: Green & group; $* *$ : & Gray Orange & roup & & SROB1 & 3.16 & 1.18 & 28.67 & 32.50 & 1.67 \\
\hline nilrar & f variatic & itree & ers Is & & SCJ1 & 3.48 & 3.26 & 31.02 & 40.05 & 2.33 \\
\hline with th & - & lies on ap & ot (Parma & ind Sharma, & $\mathrm{SCJ} 2$ & 3.96 & 2.80 & 31.07 & 39.88 & 2.00 \\
\hline 1992; Wa & ni and Mugh & nal, 2017) c & nsiderable & variation so & $\mathrm{SCJ} 3$ & 3.15 & 3.25 & 35.00 & 25.56 & 0.00 \\
\hline observe & in growth $p$ & rameterc & mainly t & & SCD1 & 3.46 & 2.81 & 32.04 & 30.04 & 3.00 \\
\hline to any tra & ining and pru & ning practic & J. & & $\mathrm{SCDH} 1$ & 3.06 & 2.04 & 33.10 & 36.85 & 0.00 \\
\hline 3.1. Folia & ge characters & & & & $\mathrm{SCDH} 2$ & 3.93 & 2.72 & 32.63 & 34.42 & 4.67 \\
\hline Leaf lengt & $\mathrm{h}$ of genotyp & es studied & om Shimla & trict varied & SCK1 & 3.15 & 1.87 & 29.54 & 27.74 & 3.33 \\
\hline from 2 & $\mathrm{~cm}$ (STB3) t & $5.56 \mathrm{~cm}$ & 5). The o & Il mean for & SCK2 & 3.37 & 1.73 & 29.78 & 21.33 & 4.00 \\
\hline leaf len & was recorde & ed as $3.53 \mathrm{c}$ & 1. Coefficie & f variation & SCK3 & 3.64 & 3.16 & 31.71 & 31.42 & 2.67 \\
\hline $\begin{array}{l}\text { was } 19 \\
\mathrm{~cm} \text { (ST }\end{array}$ & $\begin{array}{c}\% \text {. Lea } \\
\text { to } 4.4\end{array}$ & $\begin{array}{l}\text { Was } \\
\text { (SKK }\end{array}$ & $d t$ & & SCK4 & 2.94 & 3.78 & 35.62 & 29.71 & 1.00 \\
\hline $2.64 \mathrm{~cm}$. & Coefficient of & variation $\mathrm{w}$ & is recorded & 31.48\% in & SCK5 & 2.93 & 3.43 & 34.22 & 25.48 & 2.00 \\
\hline genotype & of Shimla dis & trict. Minimu & m leaf area & as observed & SCK6 & 3.05 & 3.14 & 34.15 & 27.33 & 2.67 \\
\hline & $n^{2}$ in SR3 wh & reas; max & Im was 39 . & $\mathrm{m}^{2}$ in SRB6. & SCK7 & 3.75 & 2.86 & 32.16 & 20.98 & 3.00 \\
\hline as 32.47 c & $\mathrm{m}^{2}$ and their & $\begin{array}{l}\text { genotypes } \\
\text { coefficient c }\end{array}$ & f variation & $\begin{array}{l}\text { Ict recorded } \\
\text { as observed }\end{array}$ & SM1 & 3.64 & 4.01 & 35.24 & 33.74 & 3.00 \\
\hline as $8.13 \%$. & Petiole lengt & h of genoty & es studied & nder Shimla & SM2 & 3.93 & 3.53 & 34.24 & 38.51 & 1.00 \\
\hline district va & ied from 20.9 & $8 \mathrm{~mm}$ (SCK7 & to $45.70 \mathrm{n}$ & SRB5) with & SM3 & 3.35 & 2.96 & 33.82 & 40.02 & 4.67 \\
\hline was ob & ved as 17.7 & $\%$. Gland & ere abs & . & SKK1 & 3.12 & 4.46 & 36.15 & 36.75 & 5.00 \\
\hline $\mathrm{SCJ} 3$ and & SCDh2. Numb & per of gland & in remaini & g genotypes & SKK2 & 3.64 & 1.58 & 29.89 & 37.64 & 3.00 \\
\hline ranged fr & 1.00 (SRB & 6 , SCK4 and & $\mathrm{M} 2)$ to 5.3 & (STB3) with & SKK3 & 3.53 & 2.15 & 30.05 & 30.92 & 4.67 \\
\hline $\begin{array}{l}\text { an averag } \\
\text { in Shimla }\end{array}$ & $\begin{array}{l}\text { e number of } \\
\text { district Coe }\end{array}$ & $\begin{array}{l}\text { glands } 2.94 \\
\text { fficient of }\end{array}$ & $\mathrm{n}$ the geno & pes studied & SKK4 & 3.62 & 2.48 & 30.87 & 36.38 & 5.00 \\
\hline $53.73 \%$ (T & able 3). & & & & SKUM1 & 2.89 & 2.63 & 31.88 & 30.40 & 4.67 \\
\hline Color & -5 & nyogint & ano & & SKUM2 & 2.84 & 2.97 & 32.63 & 31.54 & 5.33 \\
\hline Shim & rict & remond & e Yellow & up & SKUM3 & 3.32 & 3.06 & 36.15 & 30.71 & 3.33 \\
\hline
\end{tabular}




\begin{tabular}{lccccc}
\hline Genotype & $\begin{array}{c}\text { Leaf } \\
\text { length } \\
(\mathrm{cm})\end{array}$ & $\begin{array}{c}\text { Leaf } \\
\text { width } \\
(\mathrm{cm})\end{array}$ & $\begin{array}{c}\text { Leaf } \\
\text { area } \\
\left(\mathrm{cm}^{2}\right)\end{array}$ & $\begin{array}{c}\text { Petiole } \\
\text { length } \\
(\mathrm{mm})\end{array}$ & $\begin{array}{c}\text { No. of } \\
\text { glands }\end{array}$ \\
\hline SKUM4 & 3.51 & 3.01 & 36.11 & 29.83 & 4.67 \\
STB1 & 2.47 & 0.98 & 28.47 & 26.38 & 5.00 \\
STB2 & 2.93 & 1.25 & 30.17 & 28.45 & 5.00 \\
STB3 & 2.05 & 1.04 & 29.59 & 29.52 & 5.33 \\
Range & $2.05-$ & $0.98-$ & $26.52-$ & $20.98-$ & $0.00-$ \\
& 5.56 & 4.46 & 39.09 & 45.7 & 5.33 \\
Mean & 3.53 & 2.64 & 32.47 & 32.45 & 2.94 \\
SEm \pm & 0.11 & 0.13 & 0.41 & 0.89 & 0.24 \\
SD & 0.68 & 0.83 & 2.64 & 5.76 & 1.58 \\
CV (\%) & 19.27 & 31.48 & 8.13 & 17.77 & 53.73 \\
\hline
\end{tabular}

(147 A) in 13 genotypes; (147 B) in 16 genotypes; (146 A) in five genotypes and (146 C) in eight genotypes. Colour of matured leaves was recorded to be Green Group (138 A) in three genotypes; (138 B) in six genotypes; (138 C) in three genotypes; (137 A) in seven genotypes; (137 B) in 14 genotypes and $(137 \mathrm{C})$ in nine genotypes. Among the genotypes studied under Shimla district leaf margin was observed to be serrate in 33 genotypes and biserrate in nine genotypes. Leaf surface of all the genotypes studied under Shimla and Kinnaur district was found to be smooth (Table 4).

The variation in leaf characters are considered as distinguishing features for description and identification of fruit species and cultivars (Upshall, 1924). However, in the present study no marked variation was observed in leaf shape, surface, margins as well as colour except for some in leaf length, width and area (Table 3 and 4). The leaf area varied from 25.74 to $39.36 \mathrm{~cm}^{2}$ under genotypes of Kinnaur district whereas; in Shimla district it ranged from 26.52 to $39.09 \mathrm{~cm}^{2}$. The colour of mature leaves was green and yellow green in all the genotypes. Similar results pertaining to foliage characters were reported by various workers (Rana and Verma, 2011; Kamrani and Bouzari, 2013; Krichen et al., 2014; Ullah et al., 2017) in the past.

\subsection{Flower characters}

Initiation of flowering indicated by time of flower emergence was observed in $4^{\text {th }}$ week of February and it was extended upto $1^{\text {st }}$ week of March. Full bloom was observed from $2^{\text {nd }}$ week of March and it was extended upto $3^{\text {rd }}$ week of April in genotypes studied under Shimla district. Length of flowers in the genotypes selected for studies under Shimla district ranged from 22.89-34.43 mm. Average length of flower was observed to be $28.30 \mathrm{~mm}$ and their coefficient of variation was recorded as $9.93 \%$. Breadth of flowers in the genotypes selected for studies under Shimla district ranged from 12.21-19.12 $\mathrm{mm}$. Average breadth of flower was observed to be $16.31 \mathrm{~mm}$ and their coefficient of variation $9.72 \%$. Bearing habit in the genotypes of Shimla district was observed to be on spur (23 genotypes), on one year old shoot (11 genotypes)
Table 4: Foliage characters (non-metric) of wild apricot genotypes selected from Shimla district

\begin{tabular}{|c|c|c|c|c|}
\hline \multirow{2}{*}{$\begin{array}{l}\text { Tree } \\
\text { code }\end{array}$} & \multicolumn{2}{|c|}{ Leaf colour } & \multirow{2}{*}{$\begin{array}{l}\text { Leaf } \\
\text { margin }\end{array}$} & \multirow{2}{*}{$\begin{array}{l}\text { Leaf } \\
\text { surface }\end{array}$} \\
\hline & $\begin{array}{c}\text { Emerging } \\
\text { leaves }\end{array}$ & $\begin{array}{c}\text { Matured } \\
\text { leaves }\end{array}$ & & \\
\hline SRB1 & *YG-G 147 B & $* * \mathrm{GG}-137 \mathrm{C}$ & Serrate & Smooth \\
\hline SRB2 & YG-G $147 \mathrm{~A}$ & GG-137 C & Biserrate & Smooth \\
\hline SRB3 & YG-G $147 \mathrm{~A}$ & GG-137 B & Serrate & Smooth \\
\hline SRB4 & YG-G $146 \mathrm{C}$ & GG-137 C & Serrate & Smooth \\
\hline SRB5 & YG-G 147 B & GG-137 C & Serrate & Smooth \\
\hline SRB6 & YG-G 147 B & GG-138 C & Serrate & Smooth \\
\hline SR1 & YG-G $147 \mathrm{~A}$ & GG-138 C & Serrate & Smooth \\
\hline SR2 & YG-G $147 \mathrm{~A}$ & GG-137 C & Serrate & Smooth \\
\hline SR3 & YG-G 147 B & GG-137 B & Biserrate & Smooth \\
\hline SR4 & YG-G 147 B & GG-137 B & Serrate & Smooth \\
\hline SJM1 & YG-G 147 B & GG-137 B & Serrate & Smooth \\
\hline SJM2 & YG-G 147 B & GG-137 B & Serrate & Smooth \\
\hline SJM3 & YG-G 147 B & GG-137 B & Serrate & Smooth \\
\hline SJM4 & YG-G 147 B & GG-137 C & Serrate & Smooth \\
\hline SRoB1 & YG-G 147 B & GG-137 A & Serrate & Sm \\
\hline SCJ1 & YG-G 146 C & GG-137 A & Serrate & Smooth \\
\hline $\mathrm{SCJ} 2$ & YG-G 146 C & GG-138 B & Serrate & Smooth \\
\hline $\mathrm{SCJ} 3$ & YG-G $147 \mathrm{~A}$ & GG-138 B & Biserrate & Smooth \\
\hline SCD1 & YG-G $147 \mathrm{~A}$ & GG-137 B & Biserrate & Smooth \\
\hline SCDh1 & YG-G $147 \mathrm{~A}$ & GG-138 A & Serrate & Smooth \\
\hline SCDh2 & YG-G 147 B & GG-138 B & Serrate & Smooth \\
\hline SCK1 & YG-G 147 B & GG-138 A & Serrate & Smooth \\
\hline SCK2 & YG-G $147 \mathrm{~A}$ & GG-137 B & Serrate & Smooth \\
\hline SCK3 & YG-G 147 B & GG-137 B & Serrate & Smooth \\
\hline SCK4 & YG-G 147 A & GG-137 A & Serrate & Smooth \\
\hline SCK5 & YG-G 146 C & GG-138 B & Serrate & Smooth \\
\hline SCK6 & YG-G $146 C$ & GG-137 A & Biserrate & Smooth \\
\hline SCK7 & YG-G 146 C & GG-137 C & Serrate & Smooth \\
\hline SM1 & YG-G 147 B & GG- $137 \mathrm{C}$ & Serrate & Smooth \\
\hline SM2 & YG-G 147 B & GG-137 B & Serrate & Smooth \\
\hline SM3 & YG-G $146 \mathrm{~A}$ & GG-138 A & Serrate & Smooth \\
\hline SKK1 & YG-G 146 C & GG-138 B & Serrate & Smooth \\
\hline SKK2 & YG-G $146 \mathrm{C}$ & GG-137 C & Biserrate & Smooth \\
\hline SKK3 & YG-G $146 \mathrm{~A}$ & GG-137 A & Biserrate & Smooth \\
\hline SKK4 & YG-G 147 A & GG-138 B & Biserrate & Smooth \\
\hline SKuM1 & YG-G $146 \mathrm{~A}$ & GG-137 B & Serrate & Smooth \\
\hline SKuM2 & YG-G 147 A & GG-137 A & Serrate & Smooth \\
\hline
\end{tabular}

Table 4: Continue... 


\begin{tabular}{lcccc}
\hline \multicolumn{4}{c}{$\begin{array}{l}\text { Tree } \\
\text { code }\end{array}$} & \multicolumn{2}{c}{ Leaf colour } & Leaf & $\begin{array}{c}\text { Leaf } \\
\text { surface }\end{array}$ \\
\cline { 2 - 3 } & $\begin{array}{c}\text { Emerging } \\
\text { leaves }\end{array}$ & $\begin{array}{c}\text { Matured } \\
\text { leaves }\end{array}$ & & \\
\hline SKuM3 & YG-G 147 A & GG-137 A & Biserrate & Smooth \\
SKuM4 & YG-G 147 A & GG-138 C & Serrate & Smooth \\
STB1 & YG-G 147 A & GG-137 B & Serrate & Smooth \\
STB2 & YG-G 147 A & GG-137 B & Serrate & Smooth \\
STB3 & YG-G 147 B & GG-137 B & Serrate & Smooth \\
\hline
\end{tabular}

*: Yellow green group; **: Green group

and on both (8 genotypes). Colour of flowers was observed to be pinkish white in 31 genotypes and white in 11 genotypes among the genotypes of Shimla district (Table 5).
The variation in time of flowering may be due to the differences in the chilling hour requirement to break bud dormancy in the selected genotypes. The onset of apricot flowering is dependent on the temperature increase after dormancy and is correlated with air temperature (Blasse and Hofmann, 1993). Vachun (2003) reported that the temperature ranging from $7-9{ }^{\circ} \mathrm{C}$ determined the start of the beginning of flowering. Late blossoming is an important factor to protect any damage caused by spring frost (Unal et al., 1999), as it is one of the main objectives in the first phase of the apricot breeding programme. Such variation in flower characters have also been reported by several workers (Gulcan et al., 2006; Maria et al., 2010; Polat and Caliskan, 2013; Kumar et al., 2016).

\begin{tabular}{|c|c|c|c|c|c|c|}
\hline \multirow{2}{*}{$\begin{array}{l}\text { Geno- } \\
\text { type }\end{array}$} & \multicolumn{2}{|l|}{ Flowering time } & \multicolumn{2}{|c|}{ Size of flower } & \multirow[t]{2}{*}{ Bearing habit } & \multirow{2}{*}{$\begin{array}{l}\text { Colour of } \\
\text { flower }\end{array}$} \\
\hline & Time of opening of flower & Time of full bloom & $\begin{array}{c}\text { Length } \\
(\mathrm{mm})\end{array}$ & $\begin{array}{c}\text { Breadth } \\
(\mathrm{mm})\end{array}$ & & \\
\hline SRB1 & $4^{\text {th }}$ week of February & $2^{\text {nd }}$ week of March & 33.65 & 18.65 & One year old shoot & Pinkish white \\
\hline SRB2 & $1^{\text {st }}$ week of March & $4^{\text {th }}$ week of March & 32.87 & 17.14 & Spur & Pinkish white \\
\hline SRB3 & $4^{\text {th }}$ week of February & $2^{\text {nd }}$ week of March & 30.73 & 17.83 & Spur & Pinkish white \\
\hline SRB4 & $4^{\text {th }}$ week of February & $3^{\text {rd }}$ week of March & 29.78 & 16.56 & One year old shoot & Pinkish white \\
\hline SRB5 & $1^{\text {st }}$ week of March & $3^{\text {rd }}$ week of March & 34.43 & 19.12 & Spur & Pinkish white \\
\hline SRB6 & $1^{\text {st }}$ week of March & $3^{\text {rd }}$ week of March & 28.55 & 15.74 & Both & Pinkish white \\
\hline SR1 & $1^{\text {st }}$ week of March & $4^{\text {th }}$ week of March & 29.69 & 18.31 & Spur & while \\
\hline SR2 & $4^{\text {th }}$ week of February & $2^{\text {nd }}$ week of March & 30.11 & 17.68 & One year old shoot & Pinkish white \\
\hline SR3 & $1^{\text {st }}$ week of March & $4^{\text {th }}$ week of March & 30.19 & 16.53 & Spur & White \\
\hline SR4 & $1^{\text {st }}$ week of March & $3^{\text {rd }}$ week of March & 29.83 & 16.86 & Spur & Pinkish white \\
\hline SJM1 & $3^{\text {rd }}$ week of March & $1^{\text {st }}$ week of April & 26.57 & 14.85 & Spur & Pinkish white \\
\hline SJM2 & $2^{\text {nd }}$ week of March & $1^{\text {st }}$ week of April & 24.38 & 14.38 & Spur & Pinkish white \\
\hline SJM3 & $3^{\text {rd }}$ week of March & $1^{\text {st }}$ week of April & 28.64 & 15.17 & Spur & Pinkish white \\
\hline SJM4 & $3^{\text {rd }}$ week of March & $1^{\text {st }}$ week of April & 25.26 & 15.83 & Both & Pinkish white \\
\hline SRoB1 & $1^{\text {st }}$ week of March & $4^{\text {th }}$ Week of March & 24.85 & 14.92 & One year old shoot & White \\
\hline SCJ1 & $3^{\text {rd }}$ week of March & $2^{\text {nd }}$ week of April & 30.16 & 16.41 & One year old shoot & Pinkish white \\
\hline $\mathrm{SCJ} 2$ & $3^{\text {rd }}$ week of March & $1^{\text {st }}$ week of April & 31.09 & 16.05 & One year old shoot & Pinkish white \\
\hline $\mathrm{SCJ} 3$ & $4^{\text {th }}$ week of March & $2^{\text {nd }}$ week of April & 32.21 & 18.61 & Both & Pinkish white \\
\hline SCD1 & $3^{\text {rd }}$ week of March & $1^{\text {st }}$ week of April & 32.32 & 19.01 & Spur & Pinkish white \\
\hline SCDh1 & $3^{\text {rd }}$ week of March & $1^{\text {st }}$ week of April & 31.16 & 17.59 & Both & White \\
\hline SCDh2 & $3^{\text {rd }}$ week of March & $2^{\text {nd }}$ week of April & 30.45 & 16.83 & One year old shoot & White \\
\hline SCK1 & $1^{\text {st }}$ week of April & $3^{\text {rd }}$ week of April & 27.66 & 15.44 & Spur & Pinkish white \\
\hline SCK2 & $3^{\text {rd }}$ week of March & $1^{\text {st }}$ week of April & 28.84 & 15.07 & Spur & Pinkish white \\
\hline SCK3 & $1^{\text {st }}$ week of April & $3^{\text {rd }}$ week of April & 26.47 & 14.16 & Spur & White \\
\hline SCK4 & $1^{\text {st }}$ week of April & $3^{\text {rd }}$ week of April & 28.39 & 15.45 & Spur & White \\
\hline SCK5 & $1^{\text {st }}$ week of April & $3^{\text {rd }}$ week of April & 26.51 & 14.84 & Spur & Pinkish white \\
\hline
\end{tabular}




\begin{tabular}{|c|c|c|c|c|c|c|}
\hline \multirow{2}{*}{$\begin{array}{l}\text { Geno- } \\
\text { type }\end{array}$} & \multicolumn{2}{|l|}{ Flowering time } & \multicolumn{2}{|c|}{ Size of flower } & \multirow[t]{2}{*}{ Bearing habit } & \multirow{2}{*}{$\begin{array}{l}\text { Colour of } \\
\text { flower }\end{array}$} \\
\hline & $\begin{array}{l}\text { Time of opening of } \\
\text { flower }\end{array}$ & Time of full bloom & $\begin{array}{l}\text { Length } \\
(\mathrm{mm})\end{array}$ & $\begin{array}{l}\text { Breadth } \\
(\mathrm{mm})\end{array}$ & & \\
\hline SCK6 & $1^{\text {st }}$ week of April & $3^{\text {rd }}$ week of April & 28.06 & 16.31 & Both & Pinkish white \\
\hline SCK7 & $3^{\text {rd }}$ week of March & $1^{\text {st }}$ week of April & 29.45 & 15.19 & Both & Pinkish white \\
\hline SM1 & $3^{\text {rd }}$ week of March & $1^{\text {st }}$ week of April & 27.61 & 17.82 & One year old shoot & Pinkish white \\
\hline SM2 & $3^{\text {rd }}$ week of March & $1^{\text {st }}$ week of April & 25.55 & 16.29 & Spur & White \\
\hline SM3 & $3^{\text {rd }}$ week of March & $1^{\text {st }}$ week of April & 24.86 & 14.95 & Spur & Pinkish white \\
\hline SKK1 & $2^{\text {nd }}$ week of March & $4^{\text {th }}$ Week of March & 25.73 & 15.76 & Spur & Pinkish white \\
\hline SKK2 & $2^{\text {nd }}$ week of March & $4^{\text {th }}$ Week of March & 26.49 & 16.63 & Spur & White \\
\hline SKK3 & $2^{\text {nd }}$ week of March & $1^{\text {st }}$ week of April & 30.02 & 17.31 & Both & Pinkish white \\
\hline SKK4 & $3^{\text {rd }}$ week of March & $4^{\text {th }}$ Week of March & 25.86 & 18.05 & Spur & Pinkish white \\
\hline SKuM1 & $2^{\text {nd }}$ week of March & $4^{\text {th }}$ Week of March & 24.37 & 17.63 & One year old shoot & Pinkish white \\
\hline SKuM2 & $2^{\text {nd }}$ week of March & $4^{\text {th }}$ Week of March & 27.35 & 18.11 & One year old shoot & White \\
\hline SKuM3 & $2^{\text {nd }}$ week of March & $3 r^{d}$ week of March & 28.94 & 17.03 & Spur & Pinkish white \\
\hline SKuM4 & $2^{\text {nd }}$ week of March & $4^{\text {th }}$ Week of March & 24.44 & 13.89 & Spur & Pinkish white \\
\hline STB1 & $4^{\text {th }}$ week of February & $3^{\text {rd }}$ week of March & 22.89 & 12.21 & Both & Pinkish white \\
\hline STB2 & $4^{\text {th }}$ week of February & $2^{\text {nd }}$ week of March & 24.56 & 13.53 & One year old shoot & Pinkish white \\
\hline STB3 & $4^{\text {th }}$ week of February & $2^{\text {nd }}$ week of March & 27.84 & 15.48 & Spur & White \\
\hline Range & & & $\begin{array}{l}22.89- \\
34.43\end{array}$ & $\begin{array}{l}12.21- \\
19.12\end{array}$ & & \\
\hline Mean & & & 28.30 & 16.31 & & \\
\hline SEm \pm & & & 0.43 & 0.24 & & \\
\hline SD & & & 2.81 & 1.59 & & \\
\hline CV (\%) & & & 9.93 & 9.72 & & \\
\hline
\end{tabular}

\section{Conclusion}

The present studies were undertaken to determine some phonological, tree and leaf characters of wild apricot genotypes selected from Shimla district on the basis of fruit size and TSS. The 42 wild apricot genotypes were selected in the Shimla district for further studies. It has been determined that selected genotypes from this region showed a wide variation in most of the traits. These characters can be used for further breeding improvement of apricots like late flowering trait to protect it from spring frost.

\section{References}

Blasse, W., Hofmann, S., 1993. Phenological investigations to varieties of plum, peach and apricot. Erwerbs-Obstbau 35, 36-39.

Department of Horticulture, 2019. Horticulture development in Himachal Pradesh at a glance. www.hpagrisnet.gov. in Accessed in August, 2019.

Gulcan, R., Misirli, A., Saglam, H., Yorgancioglu, U., Erkan, S., Gumus, M., Olmez, H.A., Paydas, S., Derin, K., Eti, S., Demir, T., 2006. Properties of Turkish apricot land races.
Acta Horticulturae 701, 191-198.

Kamrani, R., Bouzari, N., 2013. Study of some Iranian apricot with leaf morphological markers (leaf characteristics). Annals of Biological Research 4, 307-311.

Krichen, L., Audergon, J.M., Neila, T.F., 2014. Variability of morphological characters among Tunisian apricot germplasm. Scientia Horticulturae 179, 328-339.

Kumar, D., Singh, D.B., Srivastava, K.K., Singh, S.R., Zargar, K.A., 2016. Performance of apricot varieties/genotypes in North Western Himalayan region of India. SAARC Journal of Agriculture 14, 107-116.

Maria, D., Cristina, P., Andreea, P., Adela, B., Viorica, B., Antonia, I., Roman, M., Alina, L., 2010. Characterization of new apricot and peach selections released from Research Station Baneasa. Journal of Horticulture, Forestry and Biotechnology 14, 65-68.

Panse, V.G., Sukhatme, P.V., 1985. Statistical Methods for Agricultural Workers. $2^{\text {nd }}$ ed. ICAR, New Delhi, India. 378.

Parmar, C., Kaushal, M.K., 1982. Prunus armeniaca L. In: Wild fruits. Kalyani Publishers, New Delhi, 66-69.

Parmar, C., Sharma, A.K., 1992. Chulli- a wild apricot from Himalayan cold desert region. Fruit Varieties Journal 
$46,35-36$.

Polat, A.A., Caliskan, O., 2013. Yield and fruit characteristics of various apricot cultivars under subtropical climate conditions of the Mediterranean region in Turkey. International Journal of Agronomy, 2013, 1-5.

Rana, J.C., Verma, V.D., 2011. Genetic resources of temperate minor fruits: indigenous and exotic. NBPGR, New Delhi, India, 2-4.

Teskey, B., Shoemaker, J.S., 1972. Tree fruit production. The AVI publishing Co. Inc. West Port Connecticut. 315.

Ullah, S., Muhammad, A., Hussian, I., Rahman, H.U., Hyder, M.Z., Din, N., 2017. Morphological variations in apricot (Prunus armeniaca) cultivars grown in Gilgit Baltistan Pakistan. Pakistan Journal of Agriculture Research 30, 1-16.

Unal, M.S., Sahin, M., Olmez, H., Celik, B., Asma, B.M., Bas,
M., 1999. The breeding of late flowering and resistant to late spring frosts apricots through crossing. Tagem/ IY/96-06-02-014, Fruit Research Institute, Malatya.

Upshall, W.F., 1924. Identification of varieties of fruit trees from leaf and other growth characters. Scientific Agriculture 4(6), 184-189.

Vachun, Z., 2003. Phenophases of blossoming and picking maturity and their relationship in twenty apricot genotypes for a period of six years. Horticultural Science 30, 43-50.

Wani, M.S., Mughal, A.H., 2017. Oil content variation and cluster analysis of different genotypes of wild apricot collected from different regions of Jammu \& Kashmir, India. International Journal of Science, Engineering and Management 2, 37-44. 